J. A. Zensus, G. B. Taylor, \& J. M. Wrobel (eds.)

\title{
3C 216: Is It the CSS Source with the Highest Rotation Measure?
}

\section{E. Lüdke}

UFSM-CCNE and LACESM-CT, Campus Universitário, 97119-900 Santa Maria RS, Brazil

W. D. Cotton

National Radio Astronomy Observatory, Charlottesville, VA 22903, U.S.A.

H. S. Sanghera

Joint Institute for VLBI in Europe, 7990 AA Dwingeloo, The Netherlands

D. Dallacasa

Istituto di Radioastronomia CNR, I-40126 Bologna, Italy

Abstract. We discuss our VLBA observations at 5 and $2.7 \mathrm{GHz}$ and our MERLIN observations at 1.6 and $5 \mathrm{GHz}$. A MERLIN+VLBA image provided good starting model for self-calibration and we obtained an unprecedent image of the bent jet of $3 \mathrm{C} 216$. Our observations suggest the detection of strong polarization position angle variation across the observing band. If this is due to Faraday rotation then $3 \mathrm{C} 216$ may have a four-figure observed rotation measure, which is unlikely to be due to errors in the polarization position angle calibration.

\section{Introduction}

The current literature on distorted extragalactic compact sources often refers to the hypothesis of light jets tunneling through a very dense and inhomogeneous medium to explain the distortion of the plasma beams and jets in compact extragalactic radio sources. Following the discovery that the superluminal jet of 3C 216 ends in a 90-degree bend (Akujor et al. 1992), the high integrated Faraday rotation $\left(R M \sim 650 \mathrm{rad} \mathrm{m}^{2}\right.$ ) seen in MERLIN polarization maps (Lüdke 1994) and the decelerating superluminal components (Barthel et al. 1988; Venturi et al. 1992) we believe that this source may be a good candidate to detect a strong jet-environment interaction and very high Faraday rotation at milliarcsecond resolution.

For this purpose we have made polarization observations with the VLBA at 5 and $2.7 \mathrm{GHz}$ to look for variations in the Faraday rotation along the jet in order to determine whether the parsec-scale jet of $3 \mathrm{C} 216$ can be tunneling through an environment with density increasing outwards.

In this paper, we present some preliminary evidence that $3 \mathrm{C} 216$ is a member of an "elite" of CSS sources with extremely-high faraday rotation (SEFR's), with four-figure rotation measures. Other high-redshift SEFR candidates are presented elsewhere in these proceedings.

\section{Discussion and Conclusions}

We were able to image the Faraday rotation along the jet of $3 \mathrm{C} 216$ based on VLBA observations with 8 mas resolution across the observing band. The fractional polarization varies by less than $2 \%$ between $\mathrm{C}$ and $\mathrm{S}$ band, implying that beam depolarization is negligible at 8 mas deconvolving beam. We also detect variations in the sign of the rotation measures at a couple of beamwidths or so 
from the core, which may be due to the effect of a disordered magnetic field structure with a tangling size scale of 20 parsecs or less $\left(\mathrm{H}_{0}=50 \mathrm{~km} / \mathrm{s} \mathrm{Mpc}^{-1}\right.$, $\left.\mathrm{q}_{0}=0.5\right)$.

If we see angular rotation towards the band, even higher Faraday rotation occurs at the jet tip since the position angle deviations are right above the jet "hook" are larger than $25^{\circ}$ between two sub-bands in the C-band observing band which are $8 \mathrm{MHz}$ apart. If it is real, 3C 216 may show the highest rotation measures observed for the CSSs. A thorough error analysis of our data is under way to confirm if this finding is real or not.

Moreover, our data certainly show that a significant excess in the amount of Faraday rotation is seen in the jet and that the low integrated Faraday rotation is due to the contribution of two regions with rotation measures having opposite signs but with absolute values of the observed rotation measures which can be even higher than the average value of $\sim 13300 \pm 80 \mathrm{rad} \mathrm{m}^{2}\left(37000 \pm 240 \mathrm{rad} \mathrm{m}^{-2}\right.$ at source co-moving frame). This result puts $3 \mathrm{C} 216$ in the "elite" CSS sources with extremely high rotation measure.

Finally we see no observational support for the idea that the jet of $3 \mathrm{C} 216$ is propagating through a gradient of increasing magnetic field and densities which would eventually disrupt the jet, since the average rotation measures do not increase by more than $30 \%$ along the bend.

Acknowledgments. E. Lüdke would like to thank the Brazilian Government/CNPq and FAPERGS for funding which enabled this research to be done and the NRAO and NFRA/JIVE for hospitality. The National Radio Astronomy Observatory is a facility of the National Science Foundation, operated under a cooperative agreement by Associated Universities, Inc.

\section{References}

Akujor C. E., et al. 1992, in Sub-Arcsecond Radio Astronomy, eds. R. J. Davis \& R. S. Booth (Cambridge: Cambridge University Press), 265-266.

Barthel P. D., Pearson T. J., \& Readhead A. C. S. 1988. ApJ, 329, L51-55.

Lüdke E. 1994. Ph.D. Thesis, Victoria University of Manchester.

Venturi, T., et al. 1994. å, 271, 65-70. 